\title{
AN ARTIFICIAL POTENTIAL FIELD BASED MOBILE ROBOT NAVIGATION METHOD TO PREVENT FROM DEADLOCK
}

\author{
Tharindu Weerakoon, Kazuo Ishii and Amir Ali Forough Nassiraei \\ Department of Human Intelligence Systems, \\ Kyushu Institute of Technology 2-4, Hibikino, Wakamatsu, Kitakyushu, 808-0196, Japan
}

\begin{abstract}
Artificial Potential Filed (APF) is the most well-known method that is used in mobile robot path planning, however, the shortcoming is that the local minima. To overcome this issue, we present a deadlock free APF based path planning algorithm for mobile robot navigation. The Proposed-APF (P-APF) algorithm searches the goal point in unknown $2 \mathrm{D}$ environments. This method is capable of escaping from deadlock and non-reachability problems of mobile robot navigation. In this method, the effective front-face obstacle information associated with the velocity direction is used to modify the Traditional APF (T-APF) algorithm. This modification solves the deadlock problem that the T-APF algorithm often converges to local minima. The proposed algorithm is explained in details and to show the effectiveness of the proposed approach, the simulation experiments were carried out in the MATLAB environment. Furthermore, the numerical analysis of the proposed approach is given to prove a deadlock free motion of the mobile robot.
\end{abstract}

\section{Introduction}

Path planning and obstacle avoidance for mobile robot navigation are challenging topics; and efficient and simple but precise algorithm is important in path planning. Both preventing any of the robot obstacle collision and guaranteeing that the robot reaches the goal are important facts when getting the robot to seek the goal.

Hwang Y.K et.al. [1] classified the path planning problems as static and dynamic depending on the environmental information available. In static problems, all the environmental information (obstacles) is known a priori the motion with no changes. In dynamic problems, no a priori environmental information is given or known partially, i.e. the visible parts of the obstacles.

In mobile robot path planning problems, various approaches such as; Visibility Graph (VG) [2],
Voronoi Diagram (VD) [3, 4], Artificial Potential Field (APF) [5-9], Virtual Force Field (VFF) [10], Virtual Force Histogram (VFH) [11-14], classical Wall-Following (WF) [15-18], Neural Networkbased approach (NN) [19, 20], Fuzzy Logic (FL) $[21,22]$ etc. are proposed in literature. These path planning algorithms can be categorized into two based on the two aspects of completeness and the scope [1, 23-28]. From the completeness point of view, algorithms can be categorized as classical or heuristic. Classical algorithms aim to find an optimal path if exists or prove that there is no solution. Heuristic algorithms try to find a better path in a short time but do not guarantee to find a solution always [1, 29]. However, the most of the classical methods are computationally expensive and heuristic methods can fail in complex environments. Depending on the scope, path planning algorithms are classified into two broad categories 


\section{Robot Path Planning (RPP) Problem}

1. Static Path Planning Problem

2. Dynamic Path Planning Problem

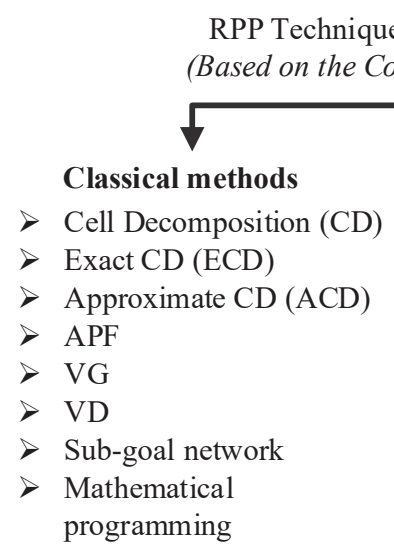

RPP Techniques Categorization - I (Based on the Completeness Property)

Figure 1. Taxonomy of mobile robot path planning algorithms
RPP Techniques Categorization - II (Based on the Scope)

\begin{tabular}{ll}
\multicolumn{1}{l}{} & Global methods \\
Local methods & $>\mathrm{CD}$ \\
$>\mathrm{APF}$ & $>\mathrm{VG}$ \\
$>\mathrm{Bug}$ & $>\mathrm{VD}$ \\
$>\mathrm{WF}$ & $>\mathrm{GA}$ \\
$>\mathrm{VFF}$ & $>\mathrm{ACO}$ \\
$>\mathrm{VFH}$ & $>\mathrm{NN}$ \\
$>\mathrm{Dynamic}$ window & $>\mathrm{PSO}$
\end{tabular}

as global path planning (deliberative paradigm) and local path planning (reactive paradigm) [1, 23-27]. A basic hierarchical classification of the path planning algorithms is given in Figure 1. The algorithms listed in Figure 1 are not tightly stick to the classification because this provides a basic categorization only; e.g. APF can also be categorized as a global path planning method. A fundamental comparison of some of the path planning methods is given in the Table 1.

Global path planning usually generates a hazard free path for the robot based on a previously known map. These methods have the completeness property which means that they are capable to find a path if it exists. Most of these methods are convergent in static environments. However, they can lose the effectiveness if an unpredicted obstacle appears in the path as a result of not including the obstacle information in the known map. Hence there is no guarantee for a collision free motion. When an unforeseen obstacle blocks the planned path, re-planning is required and it results a computationally taxing specially in unknown or dynamic environments. In addition, the complexity of the environment leads the increase of computational time of global path planning algorithms.

On the other hand, local path planning methods directly use local sensory and their basic assumption is that the robot has no a priori knowledge about the environment or partial information. Since the map of the environment is not available the entire control actions are tightly based on the perception of the robot's surrounding environment. These algorithms demand low computational effort and also the mobile robot can perceive the environmental change and decides the path in real time. Local methods are sometimes used as a component of the global planning methods or as a safety feature to avoid collision from unexpected obstacles. One disadvantage associated with the local path planning is the completeness problem.

In recent years, robotic applications have been shifting from industrial environments into some challenging scenarios like domestic applications and space/deep-sea exploration. In these settings, it is not possible to identify all the obstacles in the environment a priori to apply global path planner. Therefore, the global path planning methods are not suitable for this kind of applications. Instead, it is necessary to establish path planning methods which can handle both the discovery of new obstacle information in real-time and being fast enough to process this information to compute the path online. In contrast to any other local path planning methods, robot path planning using APF is able to consider the problem of obstacle avoidance and path planning simultaneously.

Reach to the goal while avoiding collision with the obstacles in known or unknown environments is the task of the path planning algorithm of mobile robot navigation. In that sense, in addition to the above mentioned capabilities of simultaneous path planning and obstacle avoidance, the attractive mathematical representation and concep- 
tual simplicity has made APF popular in path planning. However the APF based path planning algorithm exhibits inherit local minima problem where the robot can trap in another position away from its goal. Some methods are introduced in order to overcome this problem associated with the APF based path planning [9, 32-48].

The primary motivation behind this work is to propose a new APF based algorithm to eliminate the shortcomings of the T-APF algorithm in robot path planning. The proposed method consists of inserting the robot's motion direction and the front-face obstacle information into the APF algorithm. This creates an additional control for the robot and that will help robot to prevent from the deadlock issue.

The remainder of this paper is organized as follows. In Section 2, related work on path planning with APF is discussed. The proposed path planning method is explained in Section 3. In this section, the basic conceptual and mathematical discussion of the T-APF and P-APF methods are explained. In Section 4 , simulation experiments are discussed to show the effectiveness of the proposed algorithm over conventional method together with the results analysis and discussion. Numerical analysis to prove the deadlock escaping capability of proposed method is given in Section 5 and the conclusion in the Section 6.

\section{Related Work}

Path planning is such an important component in any mobile robot system; it is not surprising that many different approaches have been suggested in literature. In this section, we discuss briefly the APF-based path planning techniques which are proposed to solve the path planning problem.

After the APF method was being introduced by Khatib [5], many researchers made through study and a number of improved methods have been proposed to overcome its inherit shortcomings such as the local minima. There exist a large number of attempts for solving this issue. We can basically divide them into two prevailing categories of approaches depending on the technical concepts: the first is looking for better potential field instead of the T-APF by modifying or deriving new potential function (Modified/new APF approach); the second is the combined approach of APF with other techniques (Combined APF approach).

\subsection{Modified/New APF Approach}

One of the ideas is to modify the T-APF in order to escape from local minima problem and the other is to introduce a new potential function for the robot navigation.

S. S. Ge et al. [9] presented a modified repulsive potential function for path planning by taking into account the relative distance between the robot and the goal. The new repulsive force component attracts the robot towards the goal while the other component repulses the robot away from the obstacle. This work has confined the attention to solve the goal non-reachability due to obstacle nearby (GNRON) problems only.

Chen L. [31] introduced a virtual obstacle based improved APF for path planning of UUV (Unmanned Underwater Vehicle). First they determined that the UUV is trapped in local minima range, and then a virtual obstacle point was introduced. This virtual obstacle helps to change the magnitude and the direction of the artificial force and hence to escape from the local minima.

An information potential method for integrated path planning and control has been proposed by Wenjie L. et al. [32]. Their new approach uses information roadmap to escape from the local minima while increasing the probability of obtaining sensor measurements, subjected to the robot kinematics.

Doria et al. [33] reported a method inspired by the Deterministic Annealing (DA) approach to avoid local minima in APF and they have introduced a temperature parameter $(T)$ into the cost function of T-APF. The insertion of the $T$ into the cost function causes an increase of repulsion area of the obstacle and a reduction of the attraction area of the goal. DA approach is employed to avoid the random movement over the cost function surface unlike simulated annealing approach. When the robot gets stuck at a local minima point the value of the $T$ started to increase until robot escapes from the local minima point.

An improved APF based on regression search method to solve the local minima and oscillations in completely known environments has been discussed by Guanghui et al. [34]. At the same time, 
Table 1. Comparison of mobile robot path planning algorithms

\begin{tabular}{|c|c|c|}
\hline Algorithm & Advantage & Disadvantage \\
\hline APF & Real-time, $2 \mathrm{D}$ or $3 \mathrm{D}$, point/ rigid robot & Not-complete, non-optimal, local minima \\
\hline $\mathrm{CD}$ & $\begin{array}{c}\text { Complete, sound, 2D and 3D, point or rigid } \\
\text { robot }\end{array}$ & Non-optimal, Heavy computation, time \\
\hline ECD & Complete, 2D, point robot & Non-optimal, Heavy computation, time \\
\hline $\mathrm{ACD}$ & Low computation, 2D, point robot & Not-optimal, not-complete \\
\hline VG & $\begin{array}{l}\text { Complete, optimal length path, 2D or 3D, } \\
\text { point robot, static environment }\end{array}$ & $\begin{array}{l}\text { Non-optimal, heavy computation, time } \\
\text { complexity, path closer to obstacles }\end{array}$ \\
\hline VD & $\begin{array}{c}\text { Complete, safer path, 2D or arbitrary, point } \\
\text { robot }\end{array}$ & $\begin{array}{l}\text { Non-optimal, long range sensor for local path } \\
\text { planning. }\end{array}$ \\
\hline Bug & Complete, 2D, point robot & Non-optimal, long path, time complexity \\
\hline Heuristic & Less time, parallel search, point robot & Not-complete, not sound \\
\hline
\end{tabular}

algorithm finds the optimal path for the robot by connecting the sequential way points on straight lines. Local target points are introduced in order to avoid from oscillations.

An online deadlock avoidance method was presented by Chang et al. [35] for wheeled mobile robot with the presence of boxlike obstacles estimated using Hough transformation. By redefining the repulsive potential field, the local minima problem has been solved. They introduced multiobstacles into one big obstacle that cause the deadlock. This provides robot to escape from the deadlock.

An obstacle avoidance algorithm in an unknown static environment using APF has been proposed by Chunshu et al. [36]. This method was devised by combining APF with grid method and used to solve local minima problem and oscillations in static environment. The algorithm proposed herein computes the potential function value for each cell surrounding the robot cell and selects the cell with minimal value. When the robot is in the minimal value, the value of the cell is increased to escape from local minima.

Razaee H. et al. [37] introduced an adaptive APF approach for obstacle avoidance of unmanned aircrafts. The proposed potential field depends on the attitude of the aircraft and its relative position to the obstacle. They used a rotating potential field around the obstacle and simulation results were shown to prove the feasibility of the proposed method.
Random force based algorithm for local minima escape of APF approach was proposed by Lee et al. [38]. They addressed the symmetrically aligned robot-obstacle-goal situation where the deadlock takes place. When the robot is trapped at the local minima; the random force algorithm was used to escape from it.

A path planning algorithm based on the fluid mechanics was presented by Gingras D. et al. [39]. This algorithm uses the finite element method to compute a velocity potential function free from local minima. Several streamlines were computed as a road map and the optimal path was selected.

\subsection{Combined APF Approach}

Researchers have put the effort to propose verity of improvements by combining techniques for the imperfections of the T-APF algorithm.

Song et al. [40] proposed a modified potential field integrating the fuzzy control to overcome the shortcomings of the T-APF based algorithms. Repulsive force has been modified with two regulatory factors where one is affected by the distance and second is affected by the speed of the robot. Fuzzy control method was utilized to achieve the regulatory factor adjustment. One issue associated with this method is the computational time.

An Evolutionary APF combined with genetic algorithm for optimum path planning and an escape-force algorithm to escape from local minima was proposed by Vadakkepat P. et al. [41] for real time mobile robot path planning. When a local minimum is identified under certain conditions 
defined herein, an additional escape force was introduced for the algorithm.

A new real-time navigation approach by combining APF with interval type-2 Fuzzy logic system has been proposed by Melingui et al. [42]. In their work, orientation angle relative to the goal was considered to determine the probability of encountering the local minima. When the robot finds a trapping situation, fuzzy logic was used to escape from it.

Ji-Wung [43] suggested a potential field called bug potential filed which is a combination of the potential field with bug algorithm to overcome the local minima and path inefficiency problem in path planning in known environments. In this method the direction of the virtual forces to guide the robot along the path boundaries of the obstacles are determined by applying the visibility graph and the collision cone. Curvature weighted Dijkstra's shortest path algorithm was run on the visibility graph to find the optimum path.

An improved tangent bug (ITB) algorithm integrated with a potential filed to avoid from local minima issue encountered in T-APF based real-time path planning was proposed by Mohamed E.F. et al. [44]. In their method, they have introduced switching and merging conditions to guarantee a deadlock free motion. The behavior of their algorithm is classified into two modes, namely, direct motion towards the goal using APF and boundary following mode using ITB algorithm to avoid static obstacles. Switching mechanism introduced was used to overcome the local minima problem and to find the shortest path in the motion.

\section{The Proposed Path Planning Al- gorithm}

Aiming to the shortcoming of the T-APF method, an algorithm has been proposed by integrating more information into it. In this paper, we propose an APF based path planning method which helps the robot to perform a dead-lock free motion.

\subsection{Overview of the Traditional APF (T- APF)}

The APF is commonly used in path planning algorithms for autonomous mobile robots and the
APF can be treated as a landscape with several mountains generated by the obstacles and valleys where the lowest valley point represents the goal point. In the domain of robot path planning, robot is considered as a particle that moves from a high potential point via low potentials towards the goal.

APF consists of two fields: attractive field generated by the goal and repulsive field by each of the obstacles. When the robot immersed in the potential field, attractive force and repulsive force guide the robot towards the goal point. This combination of two forces is dedicated to control the motion of the robot in a safer path while keeping it away from the obstacles. Figure 2 represents the attractive potential and repulsive potential force distribution on the robot for a single obstacle existing in the environment.

\subsubsection{Attractive Force}

To simplify the path planning problem, the robot is often viewed as a mass point. The position of the robot and the goal can be expressed as vectors of $p_{r}=\left[x_{r}, y_{r}\right]^{T}$ and $p_{g}=\left[x_{g}, y_{g}\right]^{T}$ respectively. Attractive force that is produced by the goal can be expressed using the Gaussian function as in Eq. (1).

$$
F_{a t t}=a_{g}\left[1-\exp \left(-b_{g} \cdot d_{g}^{2}\right)\right] \cdot e_{g}
$$

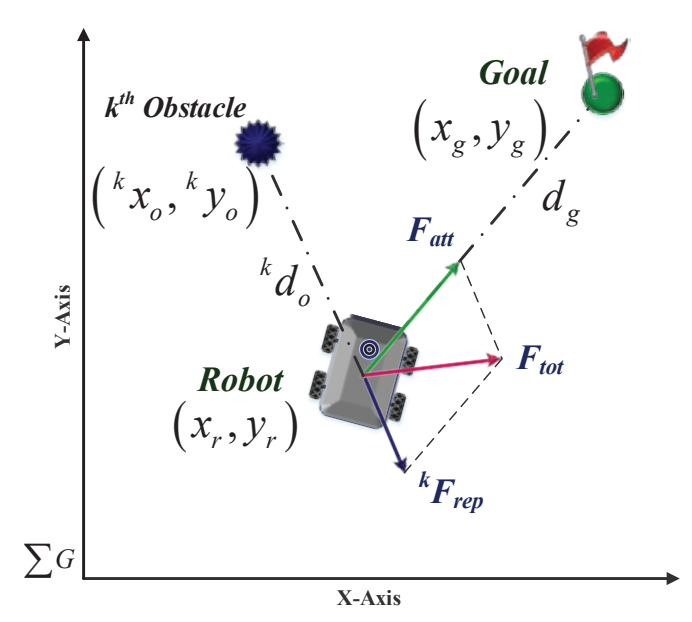

Figure 2. Force distribution of T-APF.

Where $a_{g}$ is the maximum value of the attractive force $F_{\text {att }}$ at any instance and $b_{g}$ is a constant that represents the width of the distribution. The parameter $d_{g}=\sqrt{\left(x_{r}-x_{g}\right)^{2}+\left(y_{r}-y_{g}\right)^{2}}$ is the Euclidean distance between the robot and the goal. $e_{g}$ defines 
the unit vector towards the goal point, and it can be expressed as in Eq. (2).

$$
e_{g}=\frac{1}{d_{g}}\left(\Delta x_{g} i+\Delta y_{g} j\right)
$$

Where $\Delta x_{g}=x_{g}-x_{r}$ and $\Delta y_{g}=y_{g}-y_{r}$.

\subsubsection{Repulsive Force}

The repulsive potential force generated by the $k^{\text {th }}$ obstacle can be described as in Eq. (3).

${ }^{k} F_{\text {rep }}=\left\{\begin{array}{cc}a_{o}\left[\exp \left(-b_{o} \cdot{ }^{k} d_{o}^{2}\right)\right] \cdot{ }^{k} e_{g} & \text { if }{ }^{k} d_{o} \leq d_{d} \\ 0 & \text { else }\end{array}\right.$

Where $a_{o}$ is defined as the maximum value of the repulsive force ${ }^{k} F_{\text {rep }}$ generated by the $k^{\text {th }}$ obstacle and $b_{o}$ is a constant that represents the width of the distribution. The parameter ${ }^{k} d_{o}$ is the Euclidean distance between the robot and each detected obstacle points, and $d_{d}$ is defined as the influence distance around the robot. $e_{o}$ is the unit vector towards the robot from the $k^{\text {th }}$ obstacle and it can be expressed as in Eq. (4).

$$
{ }^{k} e_{o}=\frac{1}{{ }^{k} d_{o}}\left(\Delta^{k} x_{o} i+\Delta^{k} y_{o} j\right)
$$

Where $\Delta^{k} x_{o}=x_{r}-{ }^{k} x_{o}$ and $\Delta^{k} y_{o}=y_{r}-{ }^{k} y_{o}$.

In fact, the total repulsive force can be defined as a result of the superposition of all the individual repulsive forces generated by the obstacles as in Eq. (6).

$$
F_{t o t}=F_{a t t}+\sum_{k}^{k} F_{r e p}
$$

Although the APF can perform its behavior well in path planning, it has some fatal problems. One of the main issues associated with this APF is the local minima problem which is in real called as deadlock. This happens when the total potential force becomes zero before its goal position. Therefore, this causes trapping the robot at local minima away from the goal. Deadlock can happen with the TAPF (a) collinear alignment of robot-obstacle-goal, (b) symmetrical distribution of the obstacle around the robot-goal line and (c) obstacle nearby the goal; for example.

\subsection{Proposed APF Algorithm (P-APF)}

In order to prevent from the deadlock issue associated with the T-APF based path planning algorithm a new approach is proposed. The modification is done for the repulsive force taking into account the front-face obstacle-velocity information of the robot. The new information added generates an additional controlling repulsive force to the path planning algorithm which is called obstaclevelocity repulsive force, ${ }^{k} F_{\text {rep new }}$ (from the $k^{\text {th }} \mathrm{ob-}$ stacle). The basic overview of the force distribution of proposed modification for a single obstacle case is explained in the Figure 3.

The obstacle-velocity repulsive force has a direct relationship to the angle $\delta$ between the line connects the robot-obstacle and the velocity vector, besides the distance to the obstacles. This acts perpendicular to the original repulsive force and its magnitude varies with the angle of $\delta$ (see Figure $3)$. When the robot detects an obstacle within its sensory range, a new repulsive force $\left({ }^{k} F_{\text {rep_new }}\right)$ appears in addition to the primary force. The new repulsive force component behaves to turn the robot smoothly away from the obstacles in addition to the characteristics of the original repulsive force. This behavior always leads the robot towards the goal direction only.

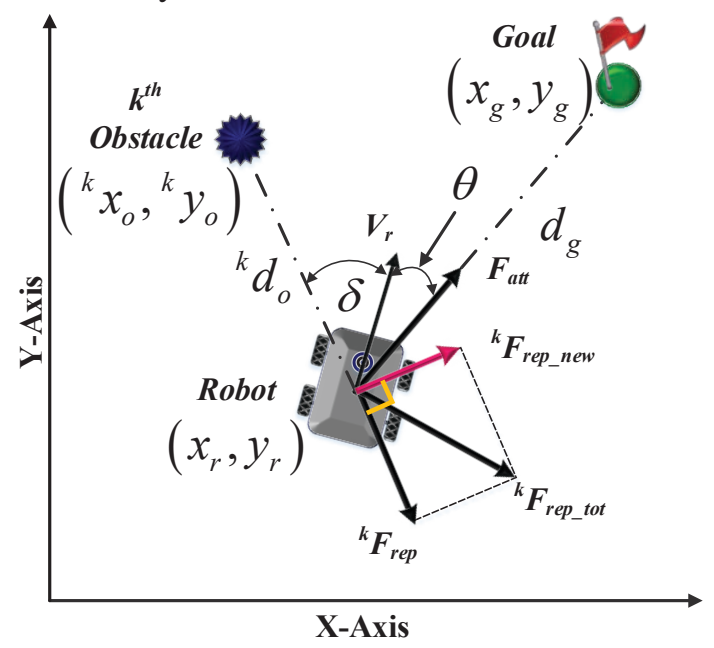

Figure 3. Overview phenomena of the P-APF and force distribution.

This characteristic of the new repulsive force always assists the robot to escape from the deadlock positions (local minima) in the motion. New repulsive force ${ }^{k} F_{\text {rep_new }}$ by the $k^{\text {th }}$ obstacle is defined as in Eq. (6) and ${ }^{k} F_{\text {rep }}$ as same as in Eq. (3). The new repulsive force consists of obstacle-velocity in- 
formation $K(\delta)$, robot turning direction towards the goal by $e_{F V}$ and the information of the basic obstacle distance. The unit vector $e_{F V}$ defined as in Eq. (7) represents the relationship between the attractive force and the robot's velocity. This will determine the direction for ${ }^{k} F_{\text {rep_new }}$ to appear either left or right side of the robot.

$$
{ }^{k} F_{\text {rep_new }}=K(\delta) \cdot\left(e_{F V} \times{ }^{k} F_{\text {rep }}\right)
$$

$$
\begin{gathered}
e_{F V}=\frac{F_{a t t} \times V_{r}}{\left\|F_{a t t}\right\|\left\|V_{r}\right\| \sin \theta} \\
K(\delta)=\frac{k_{\max }}{[1+\exp (\delta / \tau)]}
\end{gathered}
$$

The magnitude of the new repulsive force varies not only with the distance to the obstacles, but also with the angle $\delta$. The governing mathematical equation of the obstacle-velocity information $K(\delta)$ can be expressed as in Eq. (8). The variation of $K(\delta)$ with the angle $\delta$ is shown in Figure 4. The maximum value of the angle $\delta$ can be pre-defined according to our definition of the information range. As the value of $\delta$ is zero, the value of the function $K(\delta)$ has its maximum value. When $\delta$ increases, in other words, as the robot is heading away from the obstacles, the value of the $K(\delta)$ decreases exponentially and it reaches zero when $\delta=\delta_{\max }$ which is a pre-defined value ( $=120$ in this experiment). The characteristics of the function $K(\delta)$ depend on the parameter values of $k_{\max }, \tau$ and $\delta$. Those values should be properly defined in order to get the proper functionality of $K(\delta)$.

The P-APF algorithm also has the integrated capability to guide the robot properly towards the goal when there is an obstacle closer to it. As shown in the Eq. (9), when the robot-goal distance $d_{g}$ is less than the robot-obstacle distance $d_{o}$ within the obstacle detecting range $d_{d}$, robot considers only the attractive force for its motion.

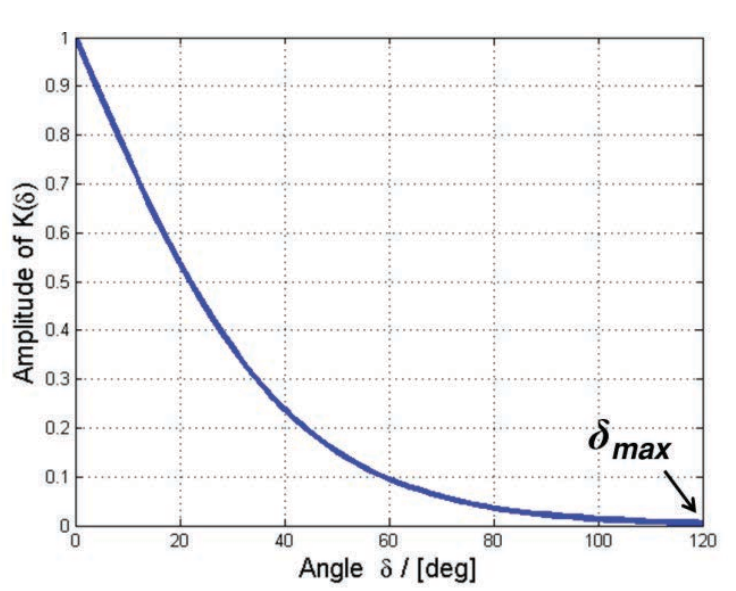

Figure 4. Variation of $K(\delta)$ with the angle $\delta$.

$$
F_{\text {tot }}=\left\{\begin{array}{cc}
F_{\text {att }}+\sum_{k}{ }^{k} F_{\text {rep }} & \text { if } \quad\left({ }^{k} d_{o} \leq d_{g} \&^{k} d_{o} \leq d_{d}\right) \\
F_{\text {att }} & \text { elseif }\left({ }^{k} d_{o}>d_{g}\right)
\end{array}\right.
$$

\subsubsection{New Repulsive Force for Solving Dead- lock Issue}

With the additional repulsive force component, the total behavior of the robot is changed. In the situation as explained in Figure 5, it is clear that the effect of the new force component makes a rotational force around the obstacle but not a radial force like in the traditional method.

Figure 5(a) shows the variation of the new repulsive force and the total repulsive force for different heading angles of the robot. When the heading direction goes away from the line connects the goal-obstacle-robot, the effect of the new repulsive force component becomes low. Similarly, for different sight angles to the obstacle, total repulsive force shows rotational characteristics as shown in Figure 5(b). As a result, new repulsive force component helps the robot to escape from the deadlock position with a smooth rotation around the obstacle.

Symmetrical distribution of the obstacles around the robot-goal line is shown in Figure 6 with force components for two different positions of the robot. As the robot moves towards the goal, the effect of the new repulsive force increases positively and keep on increasing its amplitude and being closer to the attractive force. This will help robot to reach the goal without deadlocking. But in contrast, T-APF based approach may fail when the robot passes through between the obstacles. 


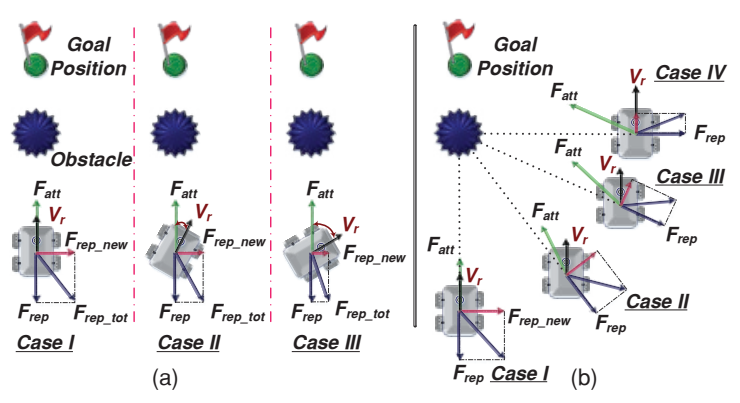

Figure 5. Rotating potential field (a) Collinear with different heading angles, (b) With different sight angles.

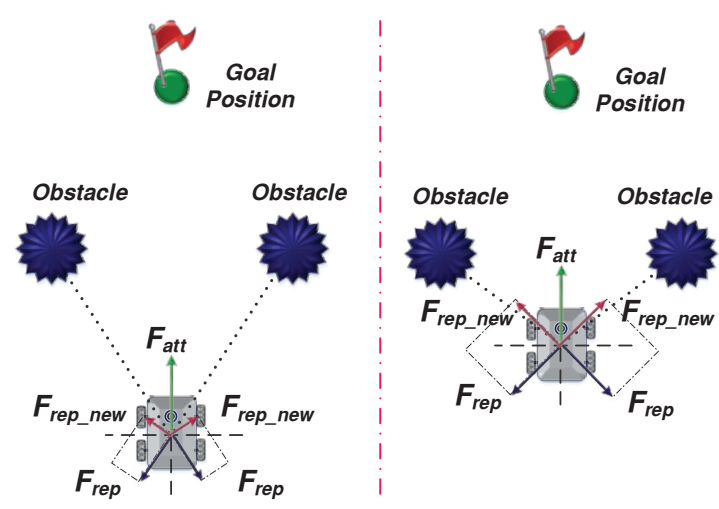

Figure 6. Symmetrical distribution of the obstacles around the robot-goal line.

\section{Simulation Experiments}

The proposed P-APF based algorithm was mathematically modeled in the MATLAB simulation environment. In order to realize the behavior of the proposed method, simulations were studied in both the static and dynamic environments under different conditions. For a better comparison of the performance of the proposed method with that of the traditional method, same parameter values of $a_{g}, a_{o}, b_{g}$ and $b_{o}$ for APF models were used. The parameters used in the attractive force and the repulsive force $a_{g}$ and $a_{o}$ as in Eq. (1) and Eq. (3) were chosen as 5 and 8 respectively for each simulation study. The parameter $b_{g}$ and $b_{o}$ were selected to be equal to 0.4 , in order to maintain a similar dis- tribution for attractive and repulsive potential fields which vanished over $4 \mathrm{~m}$ distance. The robot was considered as an omni-directional point-robot and the speed of it was chosen to be $0.05 \mathrm{~m} / \mathrm{sec}$. In dynamic environment, obstacles are rotating on the circles (radius $=4 \mathrm{~m}$ ) at the speed of $0.07 \mathrm{~m} / \mathrm{sec}$ in clockwise and counter clockwise and $0.05 \mathrm{~m} / \mathrm{sec}$ if it has a linear motion. For the simulation, it was assumed that there is a virtual LRF mounted on the robot with maximum detectable range of $4 \mathrm{~m}$ and the scanning range of 240 .

\subsection{Simulation with T-APF Algorithm}

For easy and clear explanation, at first, in both the environments the deadlock problem was studied with a single obstacle which is collinear with the goal and the robot as shown in Figure 7(a). This shows the dead-lock occurrence when an obstacle is in between the goal and the robot. For this simulation study, initially robot started to move from the point $(5,1)$, the goal is located at $(5,14)$ and there is an obstacle at $(5,8)$ on the line connects the robot and the goal. The algorithm determines the way-points of the robot's path at each step. Simulation shows that the robot is trapped around $(5,7)$ closer to the obstacle. This happens when the total potential force become zero; hence no command is generated to move the robot towards the goal which is called deadlock. Figure 7(b) explains the total potential force variation with the travelling distance of the robot in the Y-direction. It shows that the total potential force has converged to zero near a distance of $7 \mathrm{~m}$ in Y-direction.

Simulation study done for a single dynamic obstacle moving towards the robot is shown in Figure 8. Initially, robot starts to move towards the goal. As the obstacle reaches the robot, it starts to move back away from the goal with oscillations. In this unexpected motion, robot has first come to a local minimum position and since the obstacle is further moving towards the robot, the total potential force appeares and it forces the robot to move backward. As shown in Figure 8(b), total potential force become zero for a moment and as the obstacle come closer to the robot total potential force shows an oscillation near zero. This variation of total potential force towards the negative direction results the backward motion of the robot in Y-direction away from the goal. Therefore the robot never reached 
its goal position until the path of the obstacle is changed.

Another possible situation where the deadlock can happen is explained in Figure 9, which describes the symmetrical distribution of the obstacle around the robot-goal line. This takes place when the total potential force becomes zero before robot passes the obstacles. Robot has stopped around (5, 7) because the total force has reached zero as shown in Figure 9(b).
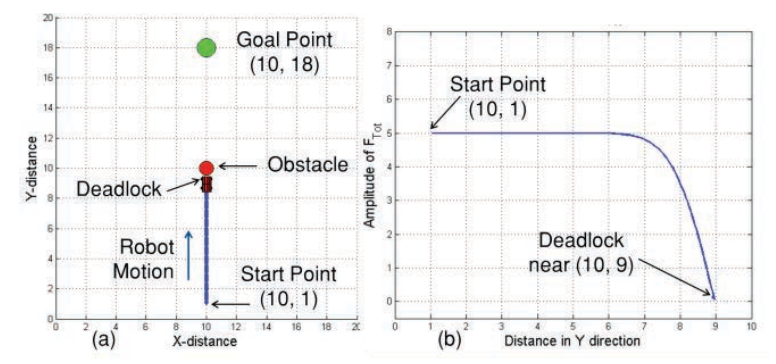

Figure 7. Deadlock issue of T-APF method for a static obstacle: (a) Simulation snapshot, (b) $F_{\text {tot }}$ variation with distance $\mathrm{Y}$.
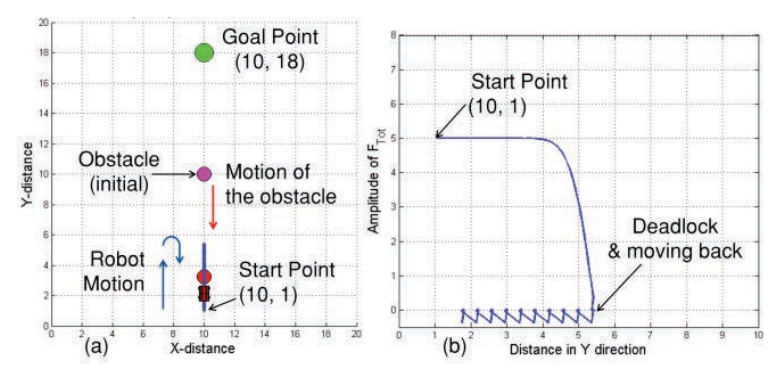

Figure 8. GNR problem of T-APF method with a moving obstacle: (a) Snapshot of the simulation, (b) $F_{\text {tot }}$ variation.
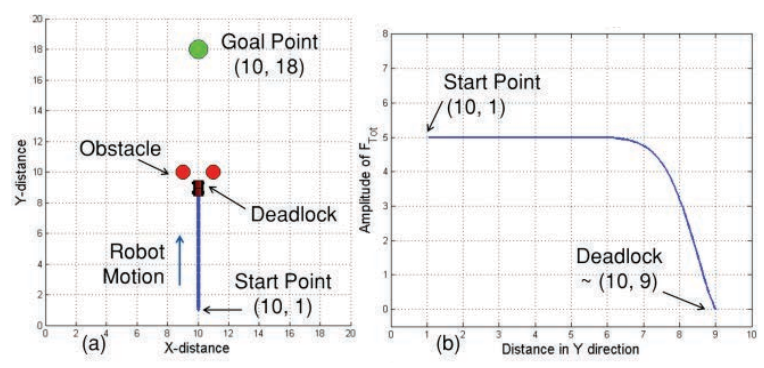

Figure 9. Deadlock issue of T-APF with symmetrical static obstacle distribution (a) Simulation snapshot, (b) $F_{t o t}$ variation with distance $\mathrm{Y}$.

\subsection{Simulation with P-APF Algorithm}

To deal with such kind of motion in dynamic environments and dead-lock problem in static environments, the P-APF algorithm gives a better solution. In order to verify the effect of the P-APF, simulation was first done in a static environment with a single obstacle and the simulation result is shown in Figure 10. The motion of the robot as in Figure 10(a) shows how it avoids the obstacle without deadlocking. Indeed with the P-APF based path planning algorithm, there is no local minimum where the total potential force becomes zero except at the goal point which causes deadlock problem. Around the point at the distance of $6 \mathrm{~m}$ in Ydirection, as in Figure 10(b), total force has reached its minimum value near but behind the obstacle before starting to increase as the robot moves further towards the obstacle. At this point, the secondary repulsive force component is acting perpendicular to the original repulsive force; hence the direction of the total potential force changes and robot starts to turn.

Figure 11 shows the simulation result carried out for a single dynamic obstacle using P-APF method. Initially, the obstacle is placed at $(5,8)$ in between the robot and the goal and its velocity towards the robot is $(0,-0.05) \mathrm{m} / \mathrm{sec}$. Robot starts to move towards the goal while the obstacle moves towards the robot. When the robot detects the obstacle, new repulsive force component helps it to go away from the direction of the obstacle and robot passes the obstacle safely and reaches the goal. As shown in the Figure 11(b), total potential force of the P-APF does not come to zero unless at the goal point.

To overcome the deadlock problem with symmetrical obstacle distribution, simulation was carried out and results are shown in Figure 12. It clearly shows the comparative advantage of P-APF over the traditional method. As the robot moves closer to the obstacles, total potential force starts to decrease but before reaching zero starts to increase because of the new repulsive force component. This happens in any condition because the new force component is acting to create a rotational force towards the goal direction only, and increasing until robot passes the obstacle (see Figure 5 (b)). 

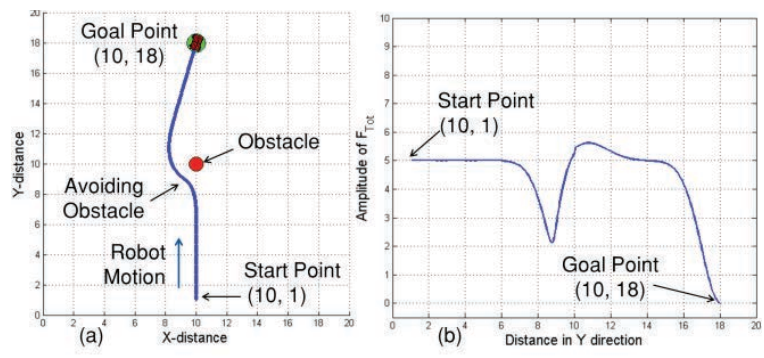

Figure 10. Simulation result of P-APF with a static obstacle.
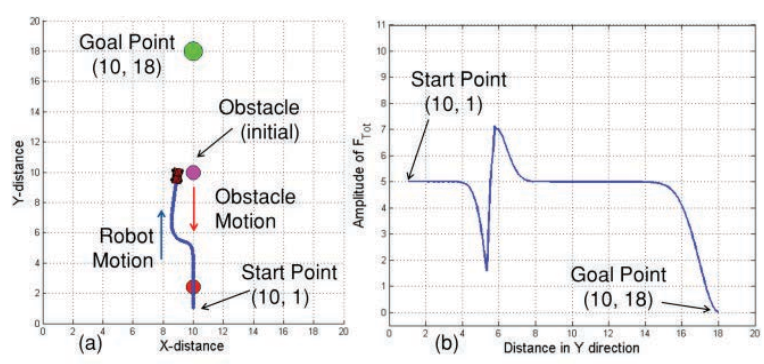

Figure 11. Simulation result of P-APF with a moving obstacle
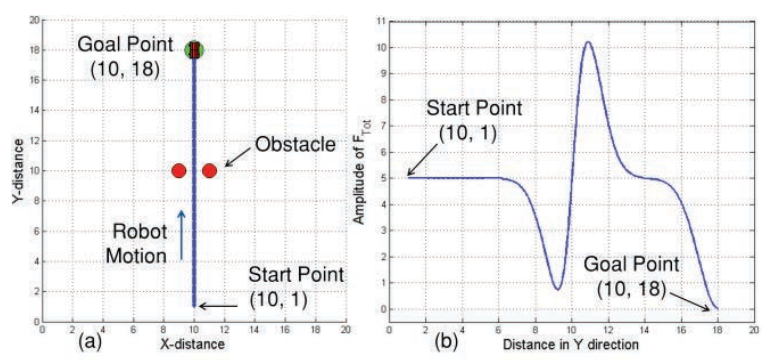

Figure 12. Simulation result of P-APF with symmetrical static obstacle distribution

\subsection{Simulation with Multi-Obstacles for $P$ - APF and T-APF}

Furthermore, for better realization of the path planning algorithm in static and dynamic multiobstacle environments, both the T-APF and P-APF algorithms were studied by performing the simulations. In both the environments, robot starts to move from the point $(5,1)$ to its goal point at $(5$, 14) for all the simulations. Figure 13(a) shows the navigation of the robot using P-APF in a static environment with many obstacles. The variation of the total potential force with respect to the distance in Y-direction is explained in Figure 13(b). Total potential force variation shows that its value becomes zero at the goal point.
Simulation study was performed for both the TAPF and P-APF in multi-obstacle dynamic environments. This study was done to verify the capability of usage of the proposed method in dynamic situation too. Figure 14 explains the simulation results for T-APF algorithm and Figure 15 explains the simulation for P-APF algorithm. Additionally, this study verifies that both the proposed and T-APFs have the capability of reaching the goal since no situation where the deadlock can happenes. However, the evaluation parameters defined to compare and evaluate the performances of the P-APF shows good performance over the traditional method (see Section 4.4).
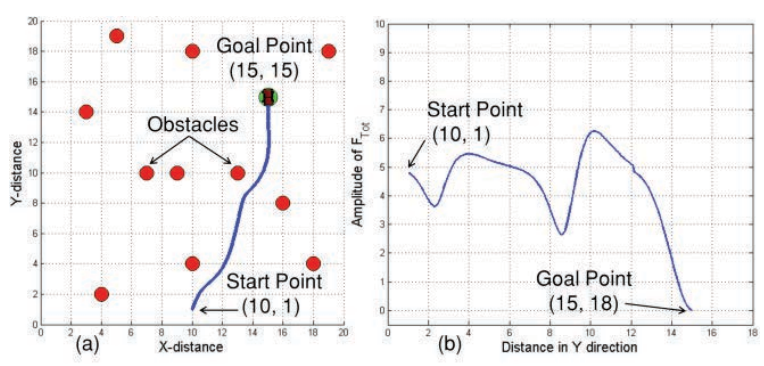

Figure 13. Simulation of P-APF method in a static environment with few obstacles.
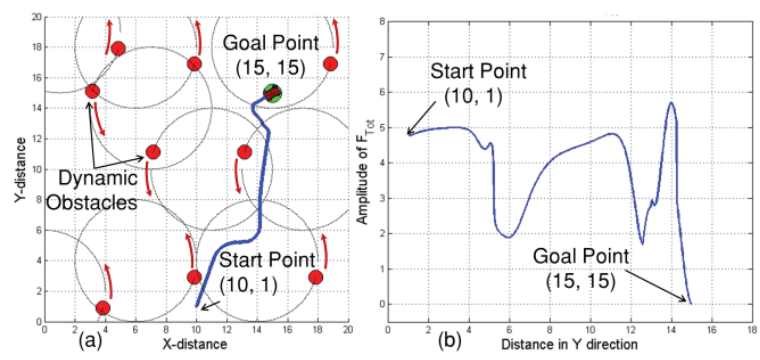

Figure 14. Simulation of T-APF method in a dynamic environment.
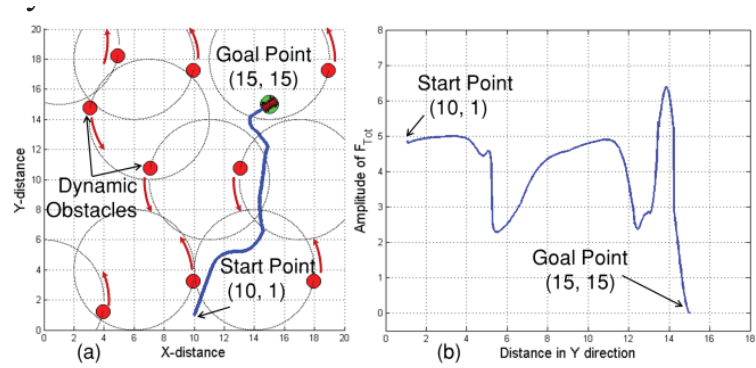

Figure 15. Simulation of P-APF method in a dynamic environment. 


\subsection{Results and Discussion}

Simulation was carried out in different environmental settings to evaluate the performance of the proposed (P-APF) path planning algorithm. Evaluation was done by comparing three parameters:

\section{$1 E_{d g}$ : Goal reachability distance error \\ $2 D_{\min }$ : Minimum clearance distance to obstacle \\ $3 D_{\text {trav }}$ : Total traveling distance}

The Table 2 shows the result of the simulation for both the T-APF and P-APF in a static environment. Simulations were carried out for single obstacle environment in a situation where the robot-obstacle-goal is aligned and for the two obstacles symmetrically distributed around the line connects the goal and the robot. But in multi-obstacles case their positions were chosen randomly. It is shown that, in single obstacle case, the robot has not reached the goal $\left(E_{d g}=9.05\right)$ with T-APF while it was able to reach the goal with P-APF. Further, navigation using P-APF has kept a considerably a large robot-to-obstacle clearance $\left(D_{\min }\right)$ in both the environments. And, it has shown that the P-APF minimizes the travelling distance too in both the static and dynamic environments. With two obstacles distributed symmetrically around the robot goal axis was also considered and evaluation parameters show that the robot has not reached the goal for T-APF. But in contrast, P-APF has prevented the deadlock and guides robot towards goal.

A comparative analysis for the evaluation parameters of the simulation for dynamic environment is given in Table 3. In both the environments, in addition to solve the deadlock issue, P-APF has maintained a considerably more $D_{\min }$ than in T-APF and minimum $D_{\text {trav }}$ all the time. By comparing the performance of both the APF approaches, P-APF shows the best performance over the traditional approach in static environments as well as in the dynamic environments.

\section{Numerical Analysis of the Pro- posed Approach}

The proposed path planning algorithm (P-APF algorithm) does not consist of local minimum which causes deadlock problem for the mobile robot path planning. Simply, local minimum point exists when collinear alignment of the robotobstacle-goal or a symmetrical distribution of the obstacles around the line which connects the robot and the goal. In those situations there is a probability to happen deadlock as the total potential becomes zero.

The T-APF and P-APF algorithms are considered to describe the deadlock scenario and the solution given by the P-APF is analyzed numerically. This analysis was done for the most critical situation of symmetrical distribution of obstacle around the robot-goal axis as shown in Figure 16. In this study, two parameters which do effect directly on deadlocking, $b_{g}$ and $b_{o}$ as in Eq. (1) and Eq. (3) and the distance between the two symmetrically distributed obstacles (obstacle gap) were considered as the variable parameters. Initially robot was placed at $(0,0)$ while the goal was at $(0,10)$. Obstacles were placed at $(5,0)$ and $(-5,5)$. By changing the gap of the obstacles symmetrically about the robotgoal line along the Y-axis, the gap was maintained in the range of $[0.2,10]$. Variation of the total repulsive force $\left(\mathbf{F}_{\mathrm{T} o t}\right)$ for both the T-APF and P-APF are shown in Figure 17. It clearly shows that the $\mathbf{F}_{\text {Tot }}$ never goes to zero except the goal point for P-APF unlike for T-APF. In some situations where the obstacles are very closely separated, $\mathbf{F}_{\mathrm{T} o t}$ of TAPF goes to negative which implies the occurrence of deadlock.

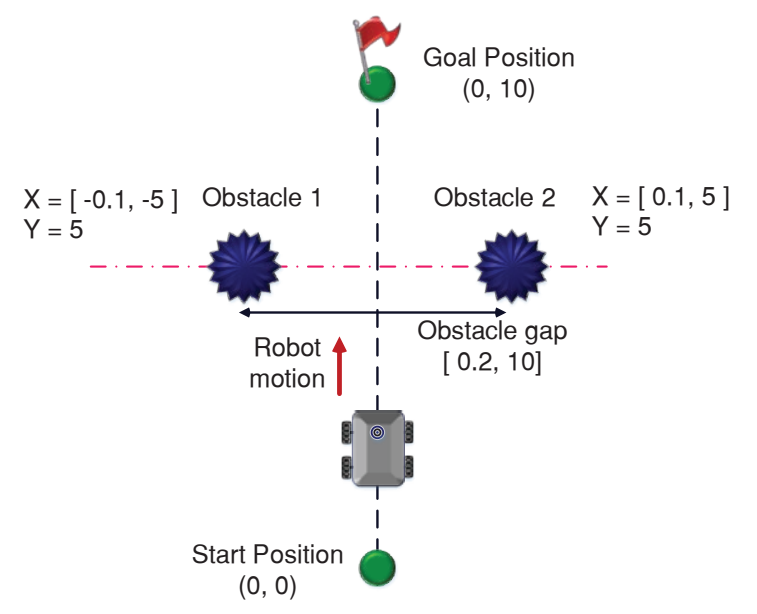

Figure 16. Simulation environment for the numerical study

The second fact of parameters $b_{g}$ and $b_{o}$ of the potential functions in Eq. (1) and Eq. (3) defines 
Table 2. Results of the simulations in static environment

\begin{tabular}{|c|c|c|c|c|c|c|}
\hline & \multicolumn{3}{|c|}{ Single Obstacle } & \multicolumn{3}{c|}{ Multi Obstacles } \\
\hline & $E_{d g}$ & $D_{\min }$ & $D_{\text {trav }}$ & $E_{d g}$ & $D_{\min }$ & $D_{\text {trav }}$ \\
\hline T-APF & 9.05 & 1.05 & N/A & $\sim 0$ & 1.37 & 15.40 \\
\hline P-APF & $\sim 0$ & 1.28 & 17.78 & $\sim 0$ & 1.41 & 15.35 \\
\hline \multicolumn{6}{|c|}{ Two Obstacles } & \\
\hline & $E_{d g}$ & $D_{\min }$ & $D_{\text {trav }}$ & & \\
\hline T-APF & 9.00 & 1.41 & N/A & & \\
\hline P-APF & $\sim 0$ & 1.00 & 17.00 & \multicolumn{1}{l}{} \\
\hline
\end{tabular}

Table 3. Results of the simulations in dynamic environment

\begin{tabular}{|c|c|c|c|c|c|c|}
\hline & \multicolumn{3}{|c|}{ Single Obstacle } & \multicolumn{3}{c|}{ Multi Obstacles } \\
\hline & $E_{d g}$ & $D_{\min }$ & $D_{\text {trav }}$ & $E_{d g}$ & $D_{\min }$ & $D_{\text {trav }}$ \\
\hline T-APF & $\sim \infty$ & 0.90 & $\sim \infty$ & $\sim 0$ & 0.91 & 17.10 \\
\hline P-APF & $\sim 0$ & 1.00 & 17.75 & $\sim 0$ & 0.93 & 17.47 \\
\hline
\end{tabular}

the potential distribution. Value of $b_{g}$ and $b_{o}$ were changed equally from 0.1 to 1 and variation $\mathbf{F}_{\mathbf{T}}$ ot for both methods is shown in Figure 18. For this study the obstacles were placed at the positions of $(1,5)$ and $(-1,5)$. It shows that for some values of $b_{g}$ and $b_{o}$, total potential force $\mathbf{F}_{\mathbf{T} o t}$ has reached zero (blue lines) where the deadlock happenes with T-APF. But, in contrast P-APF shows (red lines) no such behavior for any situation.

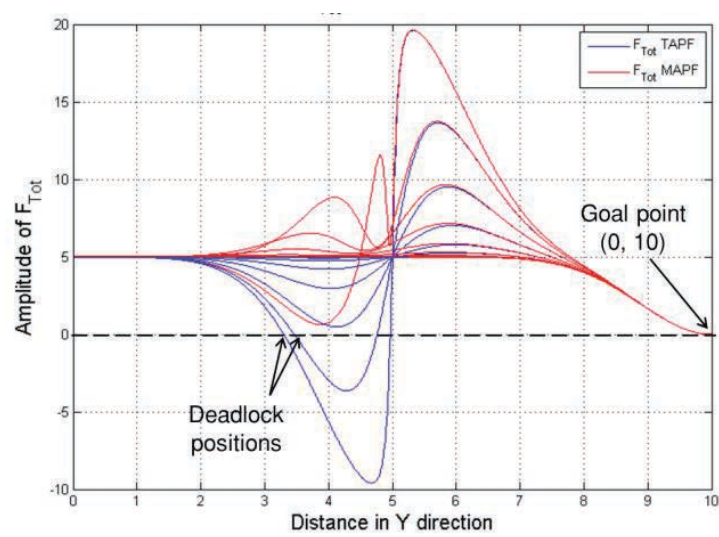

Figure 17. Variation of $\mathbf{F}_{\mathbf{T} o t}$ for different obstacle gap values

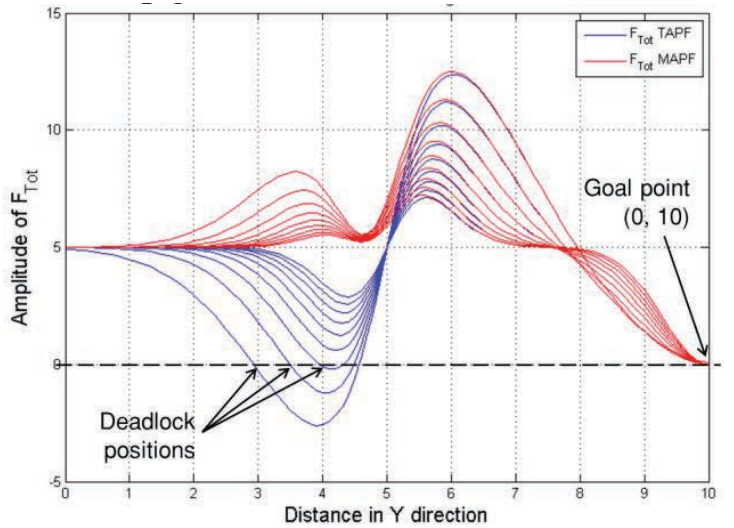

Figure 18. Variation of $\mathbf{F}_{\mathbf{T} o t}$ for different $b_{g}\left(=b_{o}\right)$ values

In an unstructured environment, P-APF can be used without any difficulty considering the geometrical parameters of the environment such as the gap between the obstacles, width of the corrido, etc. Attention is only given to the size of the robot, standard deviation of the expected potential distribution (included into the $b_{g}$ and $b_{o}$ ) and the safety distance. Overall analysis depicts that the performance of PAPF is better than that of T-APF.

\section{Conclusion}

An intelligent APF based path planning algorithm (P-APF) for mobile robot has been presented in this paper. It has the capability of escaping the lo- 
cal minima positions where deadlock happens. This study shows an efficient path planning algorithm relying only on local obstacle information, detected by LRF placed on the robot.

Comparisons have been carried out through the simulations, between this P-APF method and the TAPF method. The P-APF algorithm has proved a significant improvement on the T-APF by solving the deadlock problem well. It also produces considerable reduction of the travelling distance, maximizing the robot-obstacle clearance gap in comparison to the traditional approach. Moreover, the proposed approach provides an easy parameter calculation for the potential field distributions.

\section{References}

[1] Hwang, Y. K.; Ahuja, N., "Gross motion planning: a survey," ACM Computing Surveys (CSUR) vol. 24, no.3, 1992, pp.219-291.

[2] Sridharan, K.; Priya T. K., "A parallel algorithm for constructing reduced visibility graph and its FPGA implementation." Journal of Systems Architecture, vol. 50, no.10, 2004, pp.635-644.

[3] Bhattacharya, P.; Gavrilova, M. L., "Roadmapbased path planning-Using the Voronoi diagram for a clearance-based shortest path," Robotics \& Automation Magazine, IEEE, vol.15, no.2, 2008, pp.58-66.

[4] Garrido, S.; Moreno, L.; Abderrahim, M.; Martin, F., "Path planning for mobile robot navigation using Voronoi diagram and fast marching," Int. J. Robot. Autom. vol. 2, no.1, 2011, pp.42-64.

[5] O. Khatib, "Real-time obstacle avoidance for manipulators and mobile robots," in Proceedings of IEEE International conference on Robotics and Automation, vol. 2, Stanford, CA, March 1985, pp.500-505.

[6] Lai, L. C.; Wu, C. J.; Shiue, Y. L., “A potential field method for robot motion planning in unknown environments," Journal of the Chinese institute of engineers, vol.30, no.3, 2007, pp.369-377.

[7] J., Koren; Y. Borenstein, "Real-Time obstacle avoidance for fast mobile robot," IEEE Transaction on Systems, Man, and Cybernetics, vol. 19, no. 5, Sep/Oct 1989, pp.1179-1187.

[8] Y., Borenstein; J. Koren, "Potential field methods and their inherent limitations for mobile robot navigation," in Proceedings of the IEEE International Conference on Robotics and Automation, vol.2, 1991, pp.1398-1404.
[9] S. S. Ge; Y. J. Cui, "New Potential Functions for Mobile Robot Path Planning," IEEE Transaction on Robotics and Automation, vol. 16, no. 5, Oct. 2000, pp.615-620.

[10] Borenstein, J.; Koren, Y., "Real-time obstacle avoidance for fast mobile robots," Systems, Man and Cybernetics, IEEE Transactions on, vol.19, no.5, Sept.-Oct. 1989, pp.1179-1187.

[11] Yim, W. J.; Park, J. B. "Analysis of mobile robot navigation using vector field histogram according to the number of sectors, the robot speed and the width of the path," Control, Automation and Systems (ICCAS), $201414^{\text {th }}$ International Conference on , vol., no., 22-25 Oct. 2014, pp.1037-1040.

[12] Chaomin Luo; Yang, S.X.; Krishnan, M.; Paulik, M., "Autonomous vehicle navigation and mapping with local minima avoidance paradigm in unknown environments," World Automation Congress (WAC), 2014 , pp.823-828

[13] Jiea, D.; Xueming, M.; Kaixiang, P., "IVFH*: Real-time dynamic obstacle avoidance for mobile robots," Control Automation Robotics \& Vision (ICARCV), $201011^{\text {th }}$ International Conference on, vol., no., 7-10 Dec. 2010, pp.844-847.

[14] Bo You; Jiangyan Qiu; Dongjie Li, "A novel obstacle avoidance method for low-cost household mobile robot," Automation and Logistics, 2008. ICAL 2008. IEEE International Conference on, vol., no., 1-3 Sept. 2008, pp.111-116.

[15] Yata, T.; Kleeman, L.; Yuta, S. I., "Wall following using angle information measured by a single ultrasonic transducer," Robotics and Automation, 1998. Proceedings. 1998 IEEE International Conference on, vol.2, no., 16-20 May 1998, pp.1590-1596.

[16] Hanafi, D.; Abueejela, Y. M.; Zakaria, M. F., "Wall Follower Autonomous Robot Development Applying Fuzzy Incremental Controller," Intelligent Control and Automation, vol. 4, no.1, 2013, pp.18-25

[17] Ding, C. J.; Duan, P.; Zhang, M. L.; Han, Y. H., "Wall Following of Mobile Robot Based on Fuzzy Genetic Algorithm of Linear Interpolating,' Fuzzy Information and Engineering, vol. 2., Springer Berlin Heidelberg, 2009, pp.1579-1589.

[18] Gavrilut, I.; Tiponut, V.; Gacsadi, A.; Tepelea, L., "Wall-following method for an autonomous mobile robot using two IR sensors," WSEAS International Conference. Proceedings. Mathematics and Computers in Science and Engineering. Eds. N. E. Mastorakis, et al. No. 12. WSEAS, 2008.

[19] R. Glasis; A. Komoda; S.A.M. Gielen, "Neural network dynamics for path planning and obstalce 
avoidance," Neural Networks, vol. 8, no. 1, 1995 , pp. 125-133.

[20] C. C. Chang; K. T. Song, "Environment prediction for a mobile robot in a dynamic environment," IEEE Transaction on Robotics and Automation, vol. 13, no. 6, 1997, pp.862-872.

[21] G. Oriolo, "Real-time map building and navigation for autonomous robots in unknown environment," IEEE Transaction on Systems, Man, and Cybernetics - Part B: Cybernetics, vol. 28, no. 3, 1998, pp. 316-333.

[22] N. H. C. Yung; C. Ye, "Avoidance of moving obstacles through behavior fusion and motion prediction," IEEE Int. Conf. on Systems, Man, and Cybernetics, San Diego, CA, USA, 1998, pp. 34243429.

[23] Mohanty, P. K.; Parhi, D. R., "Controlling the motion of an autonomous mobile robot using various techniques: a review," Journal of Advance Mechanical Engineering, vol.1, no.1, 2013, pp.24-39.

[24] Lee, Gim Hee; Marcelo H. Ang Jr. "Mobile Robots Navigation, Mapping, and Localization Part I," 2009, pp.1072-1079.

[25] Hacene, N.; Mendil, B., "Autonomous Navigation and Obstacle Avoidance for a Wheeled Mobile Robots: A Hybrid Approach," International Journal of Computer Applications vol. 81, no.7, 2013, pp.34-37.

[26] Atyabi, A.; Powers, D. M., "Review of classical and heuristic-based navigation and path planning approaches," International Journal of Advancements in Computing Technology, vol. 5, no.14, 2013.

[27] Buniyamin, N.; Wan N. W. A. J.; Sariff, N.; Mohamad, Z., "A simple local path planning algorithm for autonomous mobile robots," International journal of systems applications, Engineering \& development, vol. 5, no. 2, 2011, pp.151-159.

[28] Masehian, E.; Sedighizadeh, D., "Classic and heuristic approaches in robot motion planning-a chronological review," World Academy of Science, Engineering and Technology, vol. 23, 2007, pp.101-106.

[29] Li, G.; Tamura, Y.; Yamashita, A.; Asama, H., "Effective improved artificial potential field-based regression search method for autonomous mobile robot path planning," International Journal of Mechatronics and Automation, vol. 3, no.3, 2013, pp.141-170.

[30] L. Tang; S. Dian; G. Gu; K. Zhou; S. Wang; X. Feng, "A Novel potential field method for obstacle avoidance and path planning of mobile robot," 3rd IEEE Int. Conf. on Computer Science and Technology (ICCSIT), vol. 9, no. 1, 2010, pp. 633-637.

[31] Chen, L., "UUV path planning algorithm based on virtual obstacle," Mechatronics and Automation (ICMA), 2014 IEEE International Conference on. IEEE, 2014.

[32] Lu, W.; Zhang, G.; Ferrari, S., "An Information Potential Approach to Integrated Sensor Path Planning and Control," Robotics, IEEE Transactions on, vol.30, no.4, Aug. 2014, pp.919-934

[33] Doria, N. S. F.; Freire, E. O.; Basilio, J. C., “An algorithm inspired by the deterministic annealing approach to avoid local minima in artificial potential fields," Advanced Robotics (ICAR), 2013 16th International Conference on, vol., no., 25-29 Nov. 2013, pp.1-6.

[34] Guanghui Li; Yamashita, A.; Asama, H.; Tamura, Y., "An efficient improved artificial potential field based regression search method for robot path planning," Mechatronics and Automation (ICMA), 2012 International Conference on , vol., no., 5-8 Aug. 2012, pp.1227-1232.

[35] Ya-Chun, C.; Yamamoto, Y., "Online deadlock avoidance scheme of wheeled mobile robot under the presence of boxlike obstacles," Advanced Intelligent Mechatronics. Proceedings, 2005 IEEE/ASME International Conference on, vol., no., 24-28 July 2005, pp.1535-1540.

[36] Li, C.; Cui, G.; Lu, H., "The design of an obstacle avoiding trajectory in unknown environment using potential fields," Information and Automation (ICIA), 2010 IEEE International Conference on, vol., no., 20-23 June 2010, pp.2050-2054.

[37] Rezaee, H.; Abdollahi, F., “Adaptive artificial potential field approach for obstacle avoidance of unmanned aircrafts," Advanced Intelligent Mechatronics (AIM), 2012 IEEE/ASME International Conference on, vol., no., 11-14 July 2012, pp.1-6.

[38] Lee, J.; Nam, Y.; Hong, S., "Random force based algorithm for local minima escape of potential field method," Control Automation Robotics \& Vision (ICARCV), $201011^{\text {th }}$ International Conference on. IEEE, vol., no., 7-10 Dec. 2010, pp.827832.

[39] Sugiyama, S.; Yamada, J.; Yoshikawa, T., "Path planning of a mobile robot for avoiding moving obstacles with improved velocity control by using the hydrodynamic potential," Intelligent Robots and Systems (IROS), 2010 IEEE/RSJ International Conference on, vol., no., 18-22 Oct. 2010, pp.1421-1426. 
[40] Song, Q.; \& Liu, L., "Mobile robot path planning based on dynamic fuzzy artificial potential field method," International Journal of Hybrid Information Technology vol.5, no.4, 2012, pp.85-94.

[41] Vadakkepat, P.; Tan, K. C.; Ming-Liang, W., "Evolutionary artificial potential fields and their application in real time robot path planning," Evolutionary Computation, 2000. Proceedings of the 2000 Congress on. Vol. 1. IEEE, 2000.

[42] Melingui, A.; Chettibi, T.; Merzouki, R.; Mbede, J.B., "Adaptive navigation of an omni-drive autonomous mobile robot in unstructured dynamic environments," Robotics and Biomimetics (RO-
BIO), 2013 IEEE International Conference on, vol., no., 12-14 Dec. 2013, pp.1924-1929.

[43] Ji-Wung Choi, "A potential field and bug compound navigation algorithm for nonholonomic wheeled robots," Innovative Engineering Systems (ICIES), 2012 First International Conference on, vol., no., 7-9 Dec. 2012, pp.166-171.

[44] Mohamed, E.F.; El-Metwally, K.; Hanafy, A.R., "An improved Tangent Bug method integrated with artificial potential field for multi-robot path planning," Innovations in Intelligent Systems and Applications (INISTA), 2011 International Symposium on, vol., no., 15-18 June 2011, pp.555-559.

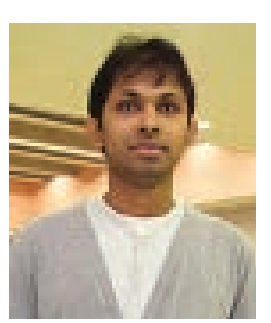

Tharindu Weerakoon was born in Sri Lanka in 1981. He received the B.Sc.Eng. (Hons) degree in Electrical and Electronic Engineering from the University of Peradeniya, Sri Lanka, in 2007 and M.Sc. degree in Robotics from Kyushu Institute of Technology, Japan in 2012. He is currently working towards the Ph.D. degree in Robotics at the Department of Human Intelligence Systems, Kyushu Institute of Technology. He was a lecturer at the Department of Electrical and Electronic Engineering, University of Peradeniya, from 2007 to 2010. His research interests include mobile robotics, path planning, SLAM.

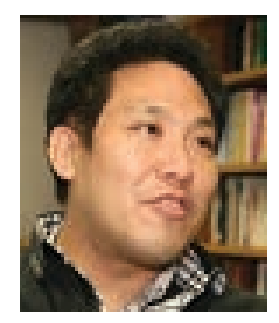

Kazuo Ishii received B.E., M.E. and $\mathrm{Ph} . \mathrm{D}$. degrees from the Department of Naval Architecture and Ocean Engineering at the University of Tokyo in 1991, 1993, and 1996, respectively. He is currently a professor at the Department of Human Intelligence Systems, Kyushu Institute of Technology. His research interests include field robotics, underwater robotics, RoboCup and its application to robotics.

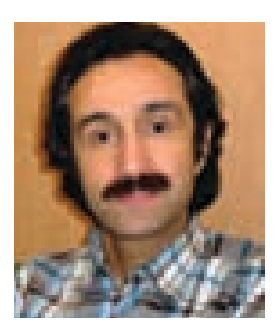

Amir Ali Forough Nassiraei was born in Mashad, Iran in 1973. He received the B.S. in Mechanical Engineering, M.S. in Bio-Mechanical Engineering from Sharif University of Technology, Iran, and Ph.D. in Robotics field from Kyushu Institute of Technology, Japan, in 1997, 2000 and 2007 , respectively. He was with GMDJapan Research Laboratory, from 2002 to 2004 and Robotics Research Institute, Japan, from 2004 to 2007. Since 2007 he has been with the Department of Human Intelligence Systems, Kyushu Institute of Technology, where he is currently an Associate Professor. His research interests include robot design, robot control and development of intelligent and practical robotic systems for social contribution. 